(2) Open Access Full Text Article

\title{
Association of Two Variable Number of Tandem Repeats in the Monoamine Oxidase A Gene Promoter with Schizophrenia
}

\author{
Takaki Tanifuji \\ Satoshi Okazaki ${ }^{1}$ \\ Ikuo Otsukal \\ Tadasu Horai' \\ Yutaka Shinko' \\ Saehyeon Kim' \\ Ichiro Sora' \\ Akitoyo Hishimoto ${ }^{1,2}$ \\ 'Department of Psychiatry, Kobe \\ University Graduate School of Medicine, \\ Kobe, Japan; ${ }^{2}$ Department of Psychiatry, \\ Yokohama City University Graduate \\ School of Medicine, Yokohama, Japan
}

Correspondence: Akitoyo Hishimoto Department of Psychiatry, Yokohama City University Graduate School of Medicine, 3-9 Fukuura, Kanazawa,

Yokohama, 236-0004, Japan

Tel +8I-45-787-2667

Fax +8I-45-783-2540

Email hishipon@yokohama-cu.ac.jp
Background: Monoamine oxidase-A (MAO-A) decomposes dopamine and serotonin, and decreased MAO-A expression increases monoamine levels and is related to the pathophysiology of schizophrenia. Previous studies have reported that variable number of tandem repeats (VNTR), namely, upstream (u)VNTR, and some single nucleotide polymorphisms (SNPs) in the MAOA gene are associated with schizophrenia.

Methods: We investigated the two VNTRs and their related SNPs (rs6323 and rs1137070) in the MAOA gene promoter in 859 patients with schizophrenia and 826 healthy controls. Distal (d)VNTR and uVNTR were genotyped with fluorescence-based fragment polymerase chain reaction assays, and rs6323 and rs1137070 with TaqMan SNP genotyping assays.

Results: Neither the genotype nor allelic frequency of the VNTRs or SNPs showed significant differences between the schizophrenia and control groups. On the other hand, analysis of the dVNTR-uVNTR-rs6323-rs1137070 haplotype showed significant association for nine repeats (9R)-3R-T-C in female patients (corrected $p=0.0006$, odds ratio [confidence interval $]=2.17[1.446-3.257])$.

Conclusion: Our findings provide novel evidence that $M A O A$ gene polymorphisms are associated with an increased risk of developing schizophrenia in females.

Keywords: haplotype, monoamine oxidase A, polymorphism, schizophrenia, variable number of tandem repeats

\section{Introduction}

Schizophrenia is a severe psychiatric disorder that affects approximately $1 \%$ of the global population. ${ }^{1}$ Schizophrenia has high heritability and is associated with complex poly genic factors. ${ }^{2}$ Pharmacological studies have indicated that a dysfunction of dopaminergic neurons could contribute to the development of schizophrenia. ${ }^{1}$ Dopamine degradation is catalyzed by monoamine oxidase (MAO) and catechol-o-methyltransferase (COMT) in the brain. ${ }^{3,4}$ Many studies have investigated the association of MAO and COMT with schizophrenia. ${ }^{4-7}$

There are two types of MAO, MAO-A and MAO-B, both of which contribute to the degradation of dopamine. ${ }^{3}$ MAO-A has primary and minor isoforms; however, the functional differences between these isoforms remain unknown. ${ }^{8}$ The $M A O A$ and $M A O B$ genes are located adjacently to each other on the $\mathrm{X}$ chromosome, in the opposite direction. ${ }^{3} \mathrm{MAO}-\mathrm{A}$ has been reported to play an important role in mental illnesses such as schizophrenia. 9,10 There is an upstream $(\mathrm{u})$ variable number of tandem repeats (VNTR) in the MAOA gene promoter. uVNTR is located $1.2 \mathrm{~kb}$ 
upstream of the $M A O A$ gene, and is present in 3 repeats (R), 3.5R, 4R, or 5R. The 3.5R and 4R are high-expression alleles, and the $2 \mathrm{R}, 3 \mathrm{R}$, and $5 \mathrm{R}$ are low-expression alleles. ${ }^{11}$ Several studies have reported that the uVNTR and its related single nucleotide polymorphisms (SNPs) of the $M A O A$ gene are associated with schizophrenia; ${ }^{12-15}$ however, these results are inconsistent with each other. $^{16-18}$

Recently, a novel VNTR, namely distal (d)VNTR has been identified in the $M A O A$ gene promoter region. dVNTR is located approximately 500 bp upstream of uVNTR and present in $8 \mathrm{R}, 9 \mathrm{R}, 10 \mathrm{R}, 11 \mathrm{R}$ or $12 \mathrm{R}$. dVNTR from $8 \mathrm{R}$ to $11 \mathrm{R}$ were found to be associated with $\mathrm{UVNTR}$, and the corresponding transcripts were evaluated. $9 \mathrm{R}$ and $10 \mathrm{R}$ are associated with the highest and lowest levels of transcription, respectively, whereas $8 \mathrm{R}$ and $11 \mathrm{R}$ show a moderate level of transcription. ${ }^{19}$ In addition, it was demonstrated that dVNTR and uVNTR are involved in the expression of the two MAO-A isoforms, wherein dVNTR increases the expression of the primary isoform that had little connection to uVNTR, and both VNTRs reduce the expression of a minor isoform that comprised a fraction of the total. ${ }^{8}$

In neuropsychiatric disorders, the combination of dVNTR and uVNTR was reported to be associated with nicotine dependence. ${ }^{20}$ However, there is no study that explored the association between the two VNTRs and other mental illnesses such as schizophrenia. In this study, we investigated the association of the two MAOA gene promoter VNTRs, and their related SNPs, with schizophrenia.

\section{Materials and Methods}

\section{Participants}

We recruited 859 patients with schizophrenia and 826 healthy controls of Japanese descent from the city of Kobe in Japan. The demographic and clinical characteristics of the participants are given in Table 1. At least two psychiatrists diagnosed every patient based on the criteria listed in the Diagnostic and Statistical Manual of Mental Disorders, 4th Edition (DSM-IV) or DSM-5, and performed unstructured interviews and reviews of the patient's medical records at each hospital. The control participants were interviewed by a psychiatrist and screened for psychiatric disorders on the basis of unstructured interviews; the inclusion criteria were not having a present, past, and family history (first degree relatives) of psychiatric disorders or substance abuse diagnosis (excluding nicotine dependence).

We implemented this study design and all related procedures in accordance with the Declaration of Helsinki. This study was approved by the Ethical Committee for Genetic Studies of Kobe University Graduate School of Medicine. Written informed consent was obtained from all the participants prior to the commencement of the experiments.

\section{Genotyping of $\mathrm{UVNTR}$ and dVNTR in the MAOA Gene Promoter}

Peripheral blood samples were drawn from the participants, and DNA was extracted using QIAamp DNA Blood Midi Kit (Qiagen Inc., Valencia, CA, USA). The quantity and purity of the DNA were assessed via NanoDrop (Thermo Fisher Scientific, Waltham, MA, USA) and the DNA samples were stored at $-80{ }^{\circ} \mathrm{C}$ until analysis. For the $M A O A$ uVNTR and dVNTR genotyping, we performed the fluorescence-based fragment polymerase chain reaction (PCR) assay according to previous studies. ${ }^{8,20}$ The MAOA uVNTR PCR assay volume $(10 \mu \mathrm{L})$ contained $1 \mathrm{ng}$ of genomic DNA, $5 \mu \mathrm{L}$ of AmpliTaq Gold Master Mix (Applied Biosystems, Foster City, CA, USA), and 15 pmol each of the following primers: 5'-GAA CGG ACG CTC CAT TCG GA-3' as a forward primer labeled with 6- Fluorescence (FAM) and 5'ACA GCC TGA CCG TGG AGA AG-3' as a reverse primer (Invitrogen, Carlsbad, CA, USA). Thermal cycling comprised $10 \mathrm{~min}$ of initial denaturing at $95{ }^{\circ} \mathrm{C}$ followed by 40 cycles of $95{ }^{\circ} \mathrm{C}$ for $30 \mathrm{~s}, 55^{\circ} \mathrm{C}$ for $30 \mathrm{~s}$, and $72{ }^{\circ} \mathrm{C}$ for $30 \mathrm{~s}$, and a final extension step of $7 \mathrm{~min}$ at $72{ }^{\circ} \mathrm{C}$. The $M A O A$ dVNTR PCR assay volume $(20 \mu \mathrm{L})$ contained $10 \mathrm{ng}$ of genomic DNA, $10 \mu \mathrm{L}$ of AmpliTaq Gold Master Mix with $10 \%$ GC enhancer (Applied Biosystems), $0.1 \mu \mathrm{M}$ of 7 deazadGTP (Bio Labs, NewYork, NY, USA) and 25 pmol of each the primers: 5'-GGG TTA AGC GCC TCA GCT TC-3' as a forward primer labeled with 6-FAM and 5'-CAA GAG TGG ACT TAA GGA AGC AG-3' as a reverse primer (Invitrogen). Thermal cycling comprised $10 \mathrm{~min}$ of initial denaturing at $95^{\circ} \mathrm{C}$ followed by 10 cycles of $95^{\circ} \mathrm{C}$ for $20 \mathrm{~s}$, the touchdown annealing steps from $65-56{ }^{\circ} \mathrm{C}$ for $20 \mathrm{~s}$, and $72{ }^{\circ} \mathrm{C}$ for $30 \mathrm{~s}, 35$ cycles of $95^{\circ} \mathrm{C}$ for $20 \mathrm{~s}, 55^{\circ} \mathrm{C}$ for $20 \mathrm{~s}$, and $72{ }^{\circ} \mathrm{C}$ for $30 \mathrm{~s}$, followed by a final extension step of $7 \mathrm{~min}$ at $72{ }^{\circ} \mathrm{C}$. Both PCR the products were analyzed using SeqStudio Genetic Analyzer (Applied Biosystems) and GeneMapper Software version 6 (Applied Biosystems). 
Table I Demographic and Clinical Characteristics of Participants

\begin{tabular}{|l|l|l|l|}
\hline & CTL (n = 826) & SCZ (n = 859) & p-value \\
\hline Sex (male/female) & $394 / 432$ & $446 / 413$ & $0.083^{\mathrm{a}}$ \\
Age/all (years), median (IQR) & $53.0(36.0,67.0)$ & $55.0(43.0,65.0)$ & $0.049^{\mathrm{b}}$ \\
Age/male, median (IQR) & $52.0(34.8,67.0)$ & $55.0(43.0,64.0)$ & $0.078^{\mathrm{b}}$ \\
Age/female, median (IQR) & $55.0(38.0,67.0)$ & $56.0(44.0,66.0)$ & $0.248^{\mathrm{b}}$ \\
Age of onset/all (years), median (IQR) & - & $24.0(20.0,30.0)$ & \\
Age of onset/male, median (IQR) & - & $23.0(19.0,30.0)$ & \\
Age of onset/female, median (IQR) & - & $25.0(20.0,31.5)$ & \\
\hline
\end{tabular}

Notes: We collected precise information about age from the clinical records of 810 (98\%) out of 826 healthy controls and 843 ( $98 \%$ ) out of 859 patients with schizophrenia, as well as about the age of onset from the clinical records of $683(80 \%)$ out of 859 patients with schizophrenia. ${ }^{a} W e$ evaluated $p$-value with the $\chi^{2}$-test between the schizophrenia and control groups. ${ }^{b} W e$ evaluated $p$-value with the Mann-Whitney $U$-test between the schizophrenia and control groups.

Abbreviations: CTL, healthy controls; SCZ, schizophrenia; IQR, interquartile range.

\section{Genotyping of rs6323 and rs II37070}

We used TaqMan SNP genotyping assays, rs6323 (Assy ID: ANEP6VZ) and rs1137070 (Assy ID:C__8878813_20), obtained from Thermo Fisher Scientific database (http:// www.thermofisher.com) as described previously. ${ }^{21}$ Genotyping was performed on a 7500 Real-Time PCR System (Applied Biosystems) according to the manufacturer's protocol.

\section{Statistical Analysis}

The data were analyzed using $\mathrm{R}$ version 4.0.0 ( $\mathrm{R}$ development core team, Vienna, Austria) and EZR version 1.42 (Saitama Medical Center, Jichi Medical University, Saitama, Japan). We used Haploview version 4.2 (Dlay Lab, Broad Institute Cambridge, MA, USA) ${ }^{22}$ to analyze allele/haplotype frequencies and genetic association in females. Differences between the groups were analyzed using $\chi^{2}$ and Mann-Whitney $U$-tests. We examined genotype-based associations, and alleles and haplotypes using the Cochran-Armitage trend test and $\chi^{2}$ _test, respectively, and permutation tests based on 10,000 replications were performed for the correction, as necessary. The threshold for statistical significance was defined as a two tailed $p<0.05$.

\section{Results}

Since the $\mathrm{X}$ chromosome contains the $M A O A$ gene, we analyzed each sex separately. We tested VNTR polymorphisms via Hardy-Weinberg equilibrium (HWE) in females with Fisher's exact test. (dVNTR, control $p=0.783$ and schizophrenia $p=0.984$; uVNTR, control $p=0.780$ and schizophrenia $p=0.108 ;$ rs6323, control $p=0.397$ and schizophrenia $p=0.606$; rs 1137070 , control $p=0.233$ and schizophrenia $p=0.584$ ). The genotype and allelic frequency for dVNTR, uVNTR, rs6323, and rs1137070 are shown in Tables 2 and 3. Neither the genotype nor allelic frequency of the VNTRs or SNPs was significantly different between the schizophrenia and control groups.

The association analysis of dVNTR-uVNTR-rs6323rs1137070 haplotype is given in Table 4. The distribution of the haplotype comprising two VNTRs and the two SNPs showed a significant association for 9R-3RT-C $(p=0.0001)$ and 10R-4R-G-T $(p=0.0139)$ in the female patients. Permutation tests based on 10,000 replications were performed, and significant differences were seen in the 9R-3R-T-C ( $p=0.0006)$ haplotype, but not in the 10R-4R-G-T $(p=0.0799)$. Participants with the 9R3R-T-C haplotype had 2.17 times increased odds of developing schizophrenia (odds ratio [confidence interval] = 2.17 [1.446-3.257], $p=0.0006$ ). The haplotype containing dVNTR(9R) showed clearly significantly differences, when analyzed using the sliding window method (Table 5).

\section{Discussion}

In this study, we investigated whether the two VNTRs (dVNTR and uVNTR) and two SNPs (rs6323 and rs1137070) in the MAOA gene promoter are associated with schizophrenia. We found that the distribution of a haplotype consisting of the two VNTRSs and two SNPs was significantly associated with schizophrenia in females.

Although multiple studies have investigated the association of uVNTR and SNPs in the MAOA gene with schizophrenia, their results are not consistent. ${ }^{12-18}$ Furthermore, no studies have investigated the association between dVNTR and schizophrenia. To the best of our knowledge, this is the first study to investigate the association of the two MAOA VNTRs with schizophrenia. 


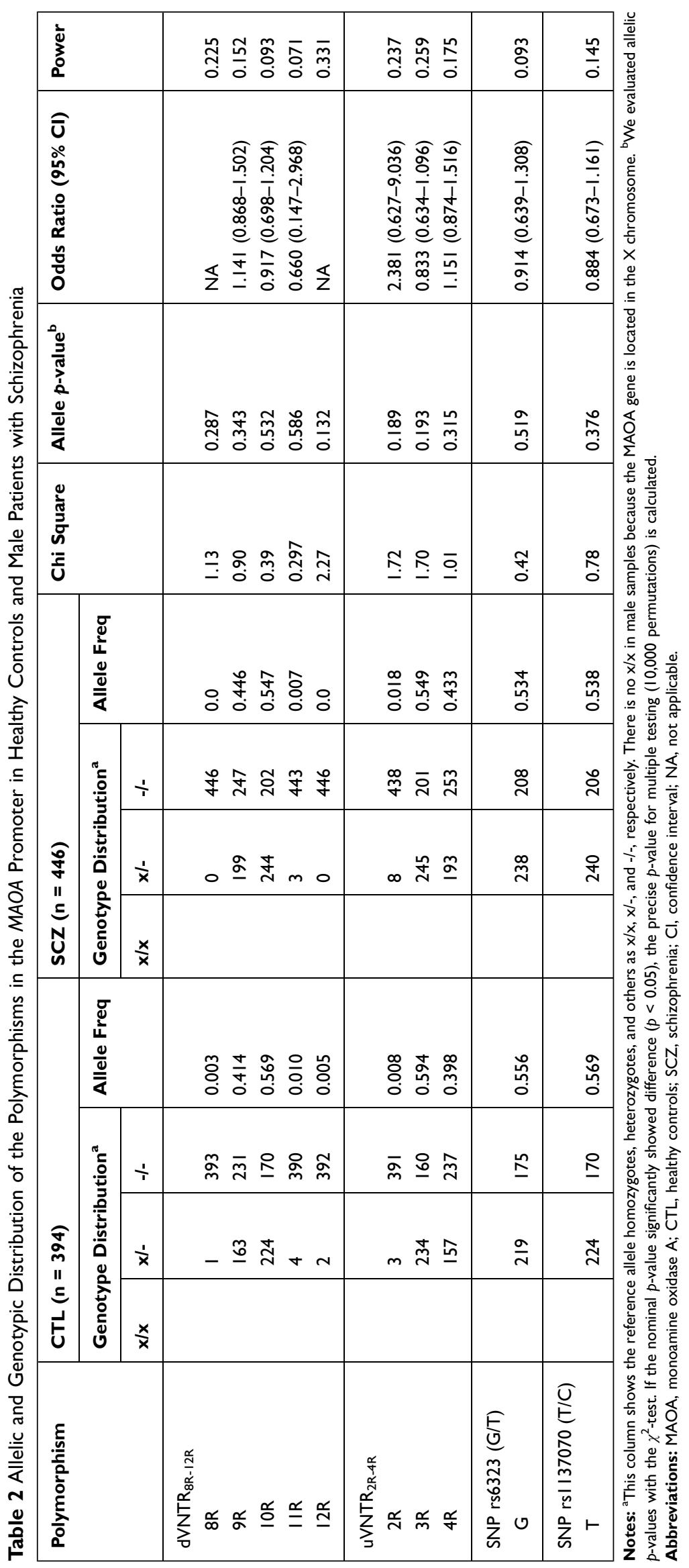




\begin{tabular}{|c|c|c|c|c|c|c|c|}
\hline \multicolumn{3}{|l|}{ 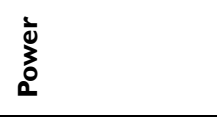 } & 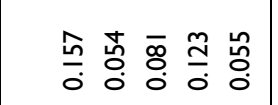 & 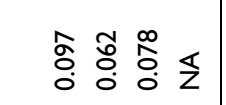 & 突 & $\overline{\check{o}}$ & 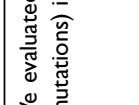 \\
\hline \multicolumn{3}{|l|}{ 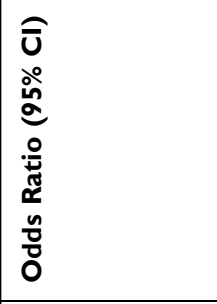 } & 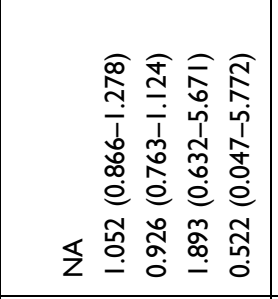 & 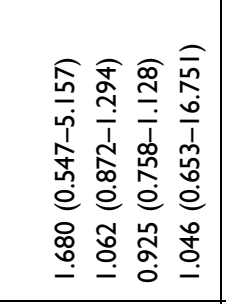 & 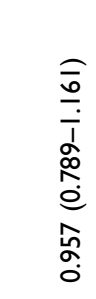 & 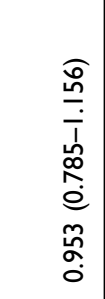 & 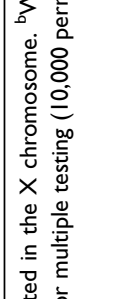 \\
\hline \multicolumn{3}{|l|}{ 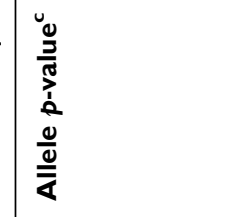 } & 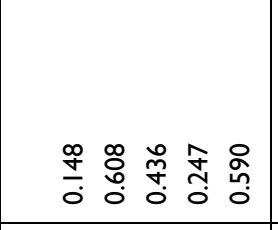 & 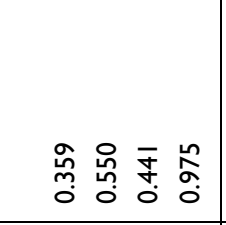 & $\begin{array}{l}\text { 峘 } \\
0\end{array}$ & డ్రి & 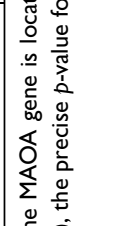 \\
\hline \multicolumn{3}{|l|}{ 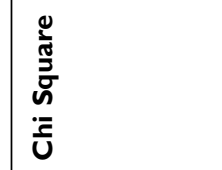 } & 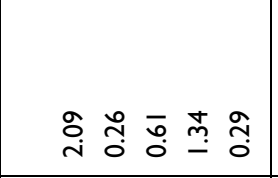 & 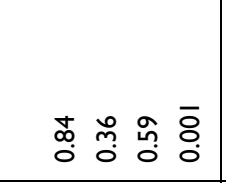 & ঙุ & $\stackrel{ \pm}{\text { ¿े }}$ & $\frac{\underline{o}}{\underline{\underline{a}}}$ \\
\hline \multicolumn{3}{|c|}{ 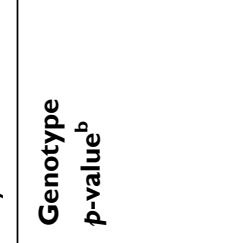 } & 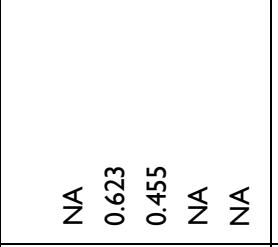 & 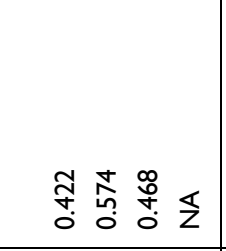 & $\begin{array}{l}\stackrel{0}{0} \\
0 \\
0\end{array}$ & $\begin{array}{l}\text { 㞷 } \\
0 \\
0\end{array}$ & \\
\hline \multicolumn{3}{|l|}{$\frac{0}{\frac{\rho}{\rho}}$} & 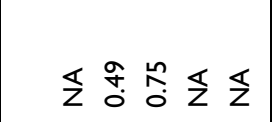 & 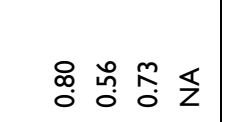 & $\stackrel{m}{0}$ & f. & \\
\hline \multirow{4}{*}{ 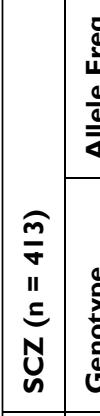 } & \multicolumn{2}{|l|}{ 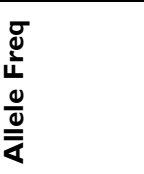 } & 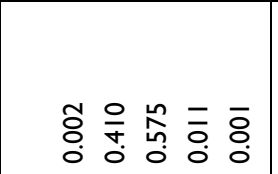 & $\begin{array}{ll}\circ \\
0 \\
0\end{array}$ & 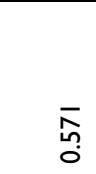 & 答 & \\
\hline & \multirow{3}{*}{ 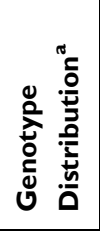 } & $\div$ & 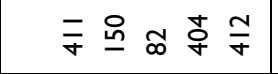 & 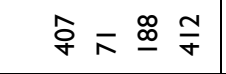 & 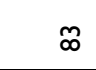 & $\widetilde{\infty}$ & \\
\hline & & $\frac{1}{x}$ & $\sim \stackrel{\widehat{\infty}}{-\underline{\infty}} \sigma-$ & 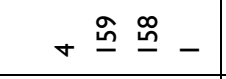 & $\stackrel{\stackrel{\infty}{ }}{\underline{-}}$ & $\underline{\underline{\omega}}$ & \\
\hline & & $\frac{x}{x}$ & 0 ○声。0 & 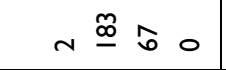 & $\underline{\mathcal{z}}$ & $\ddagger$ & \\
\hline \multicolumn{3}{|c|}{ 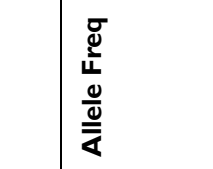 } & o 总 & 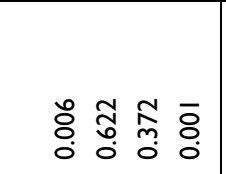 & $\begin{array}{l}\text { ô } \\
\text { ô. }\end{array}$ & 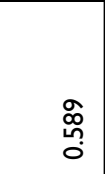 & \\
\hline \multirow{3}{*}{ 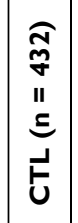 } & \multirow{3}{*}{ 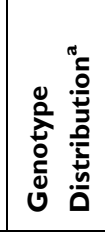 } & $\div$ & 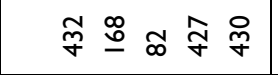 & $\overline{\hat{\gamma}} \approx \underline{\infty} \bar{\gamma}$ & $\stackrel{\infty}{\infty}$ & $\stackrel{\infty}{\infty}$ & \\
\hline & & $\dot{\dot{x}}$ & ○志 $\stackrel{\widehat{\infty}}{n}$ n $N$ & n $\stackrel{\tilde{\infty}}{\underline{\infty}}-$ & $\underline{\underline{\sigma}}$ & $\underline{\underline{0}}$ & \\
\hline & & $\frac{x}{x}$ & 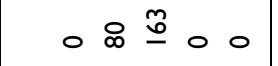 & o 10 & $\stackrel{\circ}{\varrho}$ & $\underline{\mathbf{s}}$ & \\
\hline \multicolumn{3}{|l|}{ 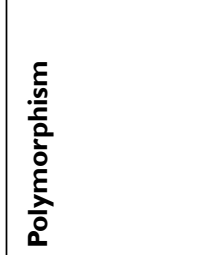 } & 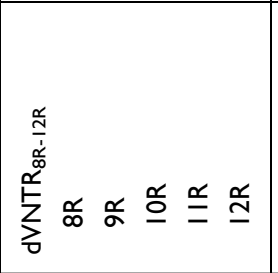 & 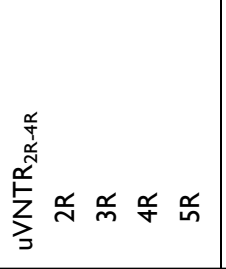 & 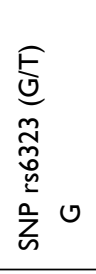 & 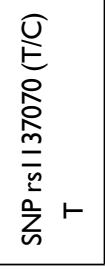 & 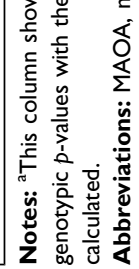 \\
\hline
\end{tabular}


Table 4 Haplotypic Distribution of Polymorphisms in the MAOA Promoter in Controls and Patients with Schizophrenia

\begin{tabular}{|c|c|c|c|c|c|c|c|c|}
\hline \multirow[t]{2}{*}{ Polymorphism } & \multicolumn{2}{|c|}{ CTL $(n=826)$} & \multicolumn{2}{|c|}{$\operatorname{SCZ}(n=859)$} & \multirow[t]{2}{*}{ Chi Square } & \multirow[t]{2}{*}{$p$-value ${ }^{a}$} & \multirow[t]{2}{*}{ Odds Ratio ( $95 \% \mathrm{Cl})$} & \multirow[t]{2}{*}{ Power } \\
\hline & $\mathbf{n}$ & Frequency & $\mathbf{n}$ & Frequency & & & & \\
\hline \multicolumn{9}{|c|}{ Male (CTL, $n=394 ; S C Z, n=446)$} \\
\hline 8R-3R-G-T & I & 0.003 & 0 & 0.00 & 1.13 & 0.287 & NA & 0.225 \\
\hline 9R-3R-T-C & 11 & 0.028 & 23 & 0.052 & 3.01 & 0.083 & $1.893(0.911-3.935)$ & 0.418 \\
\hline 9R-3R-G-T & 5 & 0.013 & 7 & 0.016 & 0.13 & $0.7 \mid 4$ & $1.24 \mid(0.39 \mid-3.940)$ & 0.054 \\
\hline $9 R-4 R-T-C$ & 142 & 0.360 & 165 & 0.370 & 0.08 & 0.774 & $1.042(0.786-1.38 I)$ & 0.048 \\
\hline 9R-4R-G-T & 1 & 0.003 & 2 & 0.004 & 0.22 & 0.637 & $1.770(0.160-19.598)$ & 0.118 \\
\hline 9R-4R-T-T & 4 & 0.010 & 2 & 0.004 & 0.95 & 0.330 & $0.439(0.080-2.4 \mathrm{II})$ & 0.189 \\
\hline IOR-2R-G-T & 3 & 0.008 & 8 & 0.018 & 1.72 & 0.189 & $2.38 \mid(0.627-9.036)$ & 0.237 \\
\hline IOR-3R-T-C & 16 & 0.041 & 12 & 0.027 & 1.22 & 0.270 & $0.653(0.305-1.398)$ & 0.204 \\
\hline IOR-3R-G-T & 195 & 0.495 & 201 & $0.45 I$ & 1.64 & 0.200 & $0.837(0.638-1.099)$ & 0.246 \\
\hline IOR-4R-T-C & I & 0.003 & 6 & 0.013 & 3.02 & 0.083 & $5.359(0.642-44.708)$ & 0.351 \\
\hline IOR-4R-G-T & 8 & 0.002 & 17 & 0.038 & 2.30 & 0.130 & $1.912(0.816-4.480)$ & 0.962 \\
\hline IOR-4R-T-T & I & 0.003 & 0 & 0.000 & 1.13 & 0.287 & NA & 0.225 \\
\hline IIR-3R-G-T & 4 & 0.010 & 2 & 0.004 & 0.95 & 0.33 & $0.439(0.080-2.412)$ & 0.189 \\
\hline IIR-4R-G-T & 0 & 0.000 & I & 0.002 & 0.88 & 0.347 & NA & 0.127 \\
\hline I2R-3R-G-T & 2 & 0.005 & 0 & 0.000 & 2.27 & 0.132 & NA & 0.331 \\
\hline \multicolumn{9}{|c|}{ Female $(C T L, n=432 ; S C Z, n=4 \mid 3)$} \\
\hline 9R-2R-T-C & I & 0.001 & 2 & 0.003 & 0.59 & 0.442 & $2.100(0.189-23.091)$ & 0.153 \\
\hline 9R-3R-T-C & 37 & 0.043 & 74 & 0.090 & 14.59 & $0.0001(0.0006)$ & $2.170(1.446-3.257)$ & 0.973 \\
\hline 9R-3R-G-T & II & 0.012 & $\mathrm{II}$ & 0.014 & 0.05 & 0.822 & I.I0I (0.475-2.554) & 0.055 \\
\hline $9 \mathrm{R}-4 \mathrm{R}-\mathrm{T}-\mathrm{C}$ & 289 & 0.336 & 244 & 0.295 & 3.19 & 0.074 & $0.829(0.674-1.018)$ & 1.000 \\
\hline 9R-4R-T-T & 3 & 0.003 & 5 & 0.006 & 0.59 & 0.444 & $1.740(0.4 \mid 4-7.303)$ & 0.151 \\
\hline 9R-4R-G-T & 3 & 0.003 & 2 & 0.003 & 0.05 & 0.822 & $0.693(0.116-4.160)$ & NA \\
\hline 9R-5R-T-C & I & 0.001 & I & 0.001 & 0.001 & 0.976 & $1.044(0.065-16.713)$ & NA \\
\hline IOR-2R-G-T & 4 & 0.005 & 6 & 0.007 & 0.36 & 0.549 & $\mathrm{I} .558(0.438-5.54 \mathrm{I})$ & 0.078 \\
\hline IOR-3R-G-T & 457 & 0.531 & 402 & 0.486 & 3.33 & 0.068 & $0.837(0.691-1.013)$ & 0.455 \\
\hline IOR-3R-T-C & 23 & 0.027 & 25 & 0.030 & 0.15 & 0.696 & 1.121 (0.632-I.987) & 0.056 \\
\hline IOR-3R G-C & I & 0.001 & 2 & 0.002 & 0.37 & $0.54 I$ & $2.085(0.189-23.037)$ & 0.078 \\
\hline IOR-4R-G-T & 19 & 0.022 & 36 & 0.044 & 6.05 & $0.0139(0.0799)$ & $2.001(1.14-3.511)$ & 0.717 \\
\hline IOR-4R-T-C & 5 & 0.005 & 4 & 0.005 & 0.03 & 0.865 & $0.832(0.223-3.110)$ & NA \\
\hline IIR-3R-G-T & 5 & 0.006 & 8 & 0.010 & 0.84 & 0.359 & $1.680(0.547-5.158)$ & 0.151 \\
\hline I2R-3R-G-T & 2 & 0.002 & I & 0.001 & 0.29 & 0.589 & $0.521(0.047-5.759)$ & 0.075 \\
\hline
\end{tabular}

Notes: ${ }^{a}$ We evaluated haplotypic $p$-values with the $\chi^{2}$-test. If the nominal $p$-value significantly showed difference $(p<0.05)$, the precise $p$-value for multiple testing ( 10,000 permutations) is calculated. The boldface indicates a significant difference.

Abbreviations: MAOA, monoamine oxidase A; CTL, healthy controls; SCZ, schizophrenia; Cl, confidence interval; NA, not applicable.

We found that the dVNTR(9R)-uVNTR(3R)-rs6323(T)rs1137070(C) haplotype was associated with schizophrenia in females. Previous studies have reported dVNTR(9R) as a high-expression allele, ${ }^{19} \mathrm{uVNTR}(3 \mathrm{R})$ as a low-expression allele, ${ }^{11}$ rs1137070(C) as a relatively high-expression allele, ${ }^{23}$ and that rs6323(T)-rs1137070(C) haplotype can lead to decreased MAO-A expression, leading to an increased risk of developing schizophrenia. ${ }^{14,15,24}$ These results including those of the current study also appear to be inconsistent. Only considering the function of each allele, and that low-expression alleles increase dopamine, which increases the risk of schizophrenia, is insufficient to clarify the molecular mechanism of schizophrenia. ${ }^{7,9,11,13,19}$ Schizophrenia is a multifactorial genetic disorder, and thus we need to investigate haplotypes with multiple variants and have a different viewpoint. ${ }^{15}$ The current study shows that several variants in the $M A O A$ locus had a combined effects on the risk of developing schizophrenia. In the similar catabolic enzyme of dopamine, the variants allele affects dopamine levels in a specific region of the brain, which is related 
Table 5 Haplotype Analysis of the MAOA Promoter in Controls and Female Patients with Schizophrenia

\begin{tabular}{|c|c|c|c|}
\hline \multirow[t]{2}{*}{ Markers } & \multicolumn{3}{|l|}{ Global $p$-value ${ }^{\text {a }} /$ Odds Ratio $(95 \% \mathrm{Cl})$} \\
\hline & Two Markers & Three Markers & Four Markers \\
\hline \multicolumn{4}{|l|}{ dVNTR (9R) } \\
\hline & $0.0004(0.0015) / 1.924(1.336-2.770)$ & & \\
\hline \multirow[t]{2}{*}{ uVNTR (3R) } & & $0.0002(0.0010) / 2.119(1.417-3.167)$ & \\
\hline & $0.0006(0.0034) / 1.778(1.273-2.483)$ & & $0.0001(0.0006) / 2.170(1.446-3.257)$ \\
\hline \multirow[t]{2}{*}{ rs6323 (T) } & & $0.0005(0.0017) / 1.808(1.293-2.530)$ & \\
\hline & $0.6620 / 1.044(0.860-1.267)$ & & \\
\hline rsII37070 (C) & & & \\
\hline
\end{tabular}

Notes: ${ }^{a}$ We evaluated haplotypic $p$-values with the $\chi^{2}$-test. If the nominal $p$-value significantly showed difference $(p<0.05)$, the precise $p$-value for multiple testing ( 10,000 permutations) is calculated. The boldface indicates a significant difference.

Abbreviations: MAOA, monoamine oxidase $\mathrm{A} ; \mathrm{Cl}$, confidence interval.

to the risk of developing schizophrenia. ${ }^{4}$ There is a possibility that low levels of MAO-A contribute to hyperfunction in the mesolimbic pathway resulting in positive symptoms of schizophrenia, whereas high levels of MAOA contribute to hypofunction in the mesocortical pathway cortex leading to negative symptoms of schizophrenia with the combined effects of variant alleles. ${ }^{5,7}$ Therefore, the 9R3R-T-C haplotype may have different functions for MAO-A expression dependent on brain regions (Figure 1). Further studies focusing on differences in the brain regions are required to determine the effects on MAO-A expression.
Indeed, previous studies have reported that uVNTR is associated with the activity of different brain regions, contributing to the developments of psychiatric disorders. ${ }^{24,25}$

It was previously reported that $\mathrm{uVNTR}$ (low-expression alleles)-rs6323(T)-rs1137070(C) haplotype is associated with schizophrenia, ${ }^{14,15}$ in line with our findings. We found that the odds ratio of the 9R-3R-T-C haplotype (corrected $p=0.0006$, odds ratio $=2.170$ ) was higher than that of the 3R-T-Chaplotype (corrected $p=0.0017$, odds ratio $=1.808$ ) (Table 5), indicating that the haplotypes containing dVNTR (9R) may increase the role of MAO-A in the pathophysiology

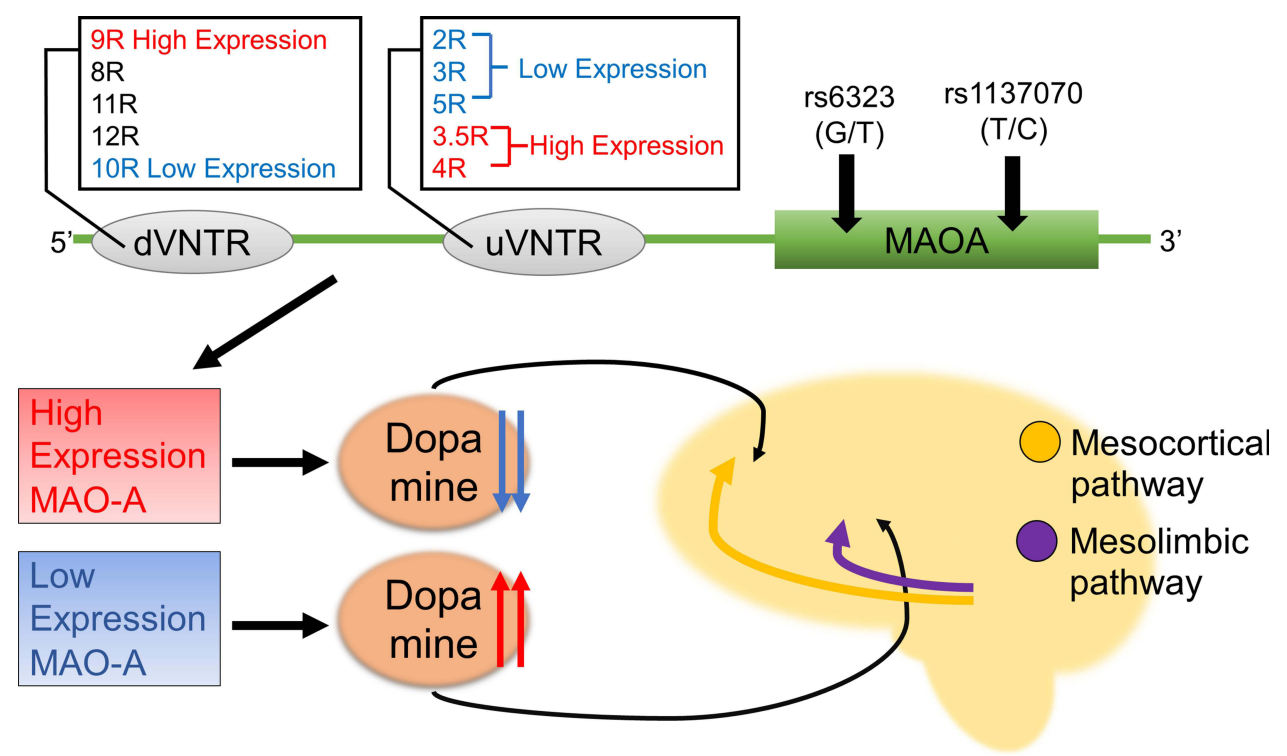

Figure I Two MAOA gene promoter VNTRs, and their related SNPs have combined effects on the pathogenesis of schizophrenia. For uVNTR, 3.5R and 4R are highexpression alleles, and the 2R, 3R, and 5R are low-expression alleles. For DVNTR, 9R and IOR are high-expression alleles, 8R and IIR are moderate expression alleles, and $I 2 R$ is unknown. Low levels of MAO-A contribute to increased dopamine levels in the mesolimbic pathway resulting in positive symptoms, and high levels of MAO-A contribute to decreased dopamine levels in the mesocortical pathway cortex leading to negative symptoms with the combined effects of two VNTRs and two SNPs. 
of schizophrenia. It is important to emphasize the combined effects that several variants had on the risk of schizophrenia and that finding new variants may help increase our current knowledge of the molecular mechanism underlying this disease.

Although recent large genome-wide association studies (GWASs) have shown that various SNPs are associated with schizophrenia, ${ }^{26-28}$ they did not include the $M A O A$ gene polymorphisms that we considered in this study. Therefore, our findings warrant reconsideration of the previous studies while also bearing in mind the effects of VNTRs and haplotypes as well as sex differences.

Our study has several limitations. First, our sample size was relatively small, and our cohort comprised participants of only Japanese descent. Further studies with large samples sizes and different populations are required to validate our findings. Second, we did not consider longitudinal effects or detailed history of the symptoms, such as whether negative or positive symptoms were dominant.

\section{Conclusion}

To the best of our knowledge, this is the first study reporting the association of the two MAOA gene promoter VNTRs, and their related SNPs with schizophrenia. Our findings show that the dVNTR(9R)-uVNTR(3R)-rs6323(T)rs1137070(C) haplotype was associated with schizophrenia in females, which may increase the risk of developing and help reveal the molecular mechanism of this disease.

\section{Acknowledgments}

We thank Makiko Ohnishi and Yasuko Nagashima for their technical assistance. This research was partly supported by JSPS KAKENHI grant numbers 15K19727, $18 \mathrm{~K} 15483$, and $21 \mathrm{~K} 07520$ (SO), as well as 26461718 , 17H04249, and 21H02852 (AH).

\section{Disclosure}

The authors report no conflicts of interest in this work.

\section{References}

1. Stepnicki P, Kondej M, Kaczor AA. Current concepts and treatments of schizophrenia. Molecules. 2018;23(8):2087. doi:10.1038/nature 13595

2. Kendler KS, Diehl SR. The genetics of schizophrenia: a current, genetic-epidemiologic perspective. Schizophr Bull. 1993;19(2): 261-285. doi:10.1093/schbul/19.2.261
3. Bortolato M, Chen K, Shih JC. Monoamine oxidase inactivation: from pathophysiology to therapeutics. Adv Drug Deliv Rev. 2008;60 (13-14):1527-1533. doi:10.1016/j.addr.2008.06.002

4. Tunbridge EM, Harrison PJ, Weinberger DR. Catechol-o-methyltransferase, cognition, and psychosis: Val158Met and beyond. Biol Psychiatry. 2006;60(2):141-151. doi:10.1016/j.biopsych.2005.10. 024

5. Schildkraut JJ, Orsulak PJ, Schatzberg AF, Herzog JM. Platelet monoamine oxidase activity in subgroups of schizophrenic disorders. Schizophr Bull. 1980;6(2):220-225. doi:10.1093/schbul/ 6.2.220

6. Meyer-Lindenberg A, Nichols T, Callicott JH, et al. Impact of complex genetic variation in COMT on human brain function. Mol Psychiatry. 2006;11(9):867-877. doi:10.1038/sj.mp.4001 860

7. Berger PA, Ginsburg RA, Barchas JD, Murphy DL, Wyatt RJ. Platelet monoamine oxidase in chronic schizophrenic patients. Am J Psychiatry. 1978;135(1):95-99. doi:10.1176/ajp.135.1.95

8. Manca M, Pessoa V, Lopez AI, et al. The regulation of monoamine oxidase a gene expression by distinct variable number tandem repeats. J Mol Neurosci. 2018;64(3):459-470. doi:10.1007/s12031018-1044-z

9. Meyer JH, Ginovart N, Boovariwala A, et al. Elevated monoamine oxidase a levels in the brain: an explanation for the monoamine imbalance of major depression. Arch Gen Psychiatry. 2006;63 (11):1209-1216. doi:10.1001/archpsyc.63.11.1209

10. Ziegler C, Domschke K. Epigenetic signature of MAOA and MAOB genes in mental disorders. J Neural Transm (Vienna). 2018;125 (11):1581-1588. doi:10.1007/s00702-018-1929-6

11. Sabol SZ, Hu S, Hamer D. A functional polymorphism in the monoamine oxidase A gene promoter. Hum Genet. 1998;103(3):273-279. doi: $10.1007 / \mathrm{s} 004390050816$

12. Culej J, Nikolac Gabaj N, Stefanovic M, Karlovic D. Prediction of schizophrenia using MAOA-uVNTR polymorphism: a case-control study. Indian J Psychiatry. 2020;62(1):80-86. doi:10.4103/psychiatry.IndianJPsychiatry_54_19

13. Jönsson EG, Norton N, Forslund K, et al. Association between a promoter variant in the monoamine oxidase A gene and schizophrenia. Schizophr Res. 2003;61(1):31-37. doi:10.1016/s0920-9964(02)00224-4

14. Qiu HT, Meng HQ, Song C, et al. Association between monoamine oxidase (MAO)-A gene variants and schizophrenia in a Chinese population. Brain Res. 2009;1287:67-73. doi:10.1016/j.brainres. 2009.06.072

15. Sun Y, Zhang J, Yuan Y, Yu X, Shen Y, Xu Q. Study of a possible role of the monoamine oxidase A (MAOA) gene in paranoid schizophrenia among a Chinese population. Am J Med Genet B Neuropsychiatr Genet. 2012;159B(1):104-111. doi:10.1002/ajmg.b.32009

16. Li D, He L. Meta-study on association between the monoamine oxidase A gene (MAOA) and schizophrenia. Am J Med Genet B Neuropsychiatr Genet. 2008;147B(2):174-178. doi:10.1002/ajmg.b.30570

17. Sasaki T, Hattori M, Sakai T, et al. The monoamine oxidase-a gene and major psychosis in japanese subjects. Biol Psychiatry. 1998;44 (9):922-924. doi:10.1016/S0006-3223(97)00522-2

18. Syagailo YV, Stöber G, Grässle M, et al. Association analysis of the functional monoamine oxidase A gene promoter polymorphism in psychiatric disorders. Am J Med Genet B Neuropsychiatr Genet. 2001;105(2):168-171. doi:10.1002/ajmg.1193

19. Philibert RA, Wernett P, Plume J, Packer H, Brody GH, Beach SR. Gene environment interactions with a novel variable Monoamine Oxidase A transcriptional enhancer are associated with antisocial personality disorder. Biol Psychol. 2011;87(3):366-371. doi:10.10 16/j.biopsycho.2011.04.007

20. Koks G, Prans E, Ho XD, et al. Genetic interaction between two VNTRs in the MAOA gene is associated with the nicotine dependence. Exp Biol Med (Maywood). 2020;245(8):733-739. doi: $10.1177 / 1535370220916888$ 
21. Okazaki S, Hishimoto A, Otsuka I, et al. Increased serum levels and promoter polymorphisms of macrophage migration inhibitory factor in schizophrenia. Prog Neuropsychopharmacol Biol Psychiatry. 2018;83:33-41. doi:10.1016/j.pnpbp.2018.01.001

22. Barrett JC, Fry B, Maller J, Daly MJ. Haploview: analysis and visualization of LD and haplotype maps. Bioinformatics. 2005;21 (2):263-265. doi:10.1093/bioinformatics/bth457

23. Hotamisligil GS, Breakefield XO. Human monoamine oxidase A gene determines levels of enzyme activity. Am J Hum Genet. 1991;49:383-392.

24. Zhang J, Chen Y, Zhang K, et al. A cis-phase interaction study of genetic variants within the MAOA gene in major depressive disorder. Biol Psychiatry. 2010;68(9):795-800. doi:10.1016/j.biopsych.2010.06.004

25. Lee B-T, Ham B-J. Monoamine oxidase A-uVNTR genotype affects limbic brain activity in response to affective facial stimuli. Neuroreport 2008;19(5):515-519. doi:10.1097/WNR.0b013e3282f94294
26. Ikeda M, Takahashi A, Kamatani Y, et al. Genome-wide association study detected novel susceptibility genes for schizophrenia and shared trans-populations/diseases genetic effect. Schizophr Bull. 2019;45(4):824-834. doi:10.1093/schbul/sby140

27. Lam M, Chen CY, Li Z, et al. Comparative genetic architectures of schizophrenia in East Asian and European populations. Nat Genet. 2019;51(12):1670-1678. doi:10.1038/s41588-019-0512-x

28. Schizophrenia Working Group of the Psychiatric Genomics C. Biological insights from 108 schizophrenia-associated genetic loci. Nature. 2014;511(7510):421-427. doi:10.1038/ nature 13595.
Neuropsychiatric Disease and Treatment

\section{Publish your work in this journal}

Neuropsychiatric Disease and Treatment is an international, peerreviewed journal of clinical therapeutics and pharmacology focusing on concise rapid reporting of clinical or pre-clinical studies on a range of neuropsychiatric and neurological disorders. This journal is indexed on PubMed Central, the 'PsycINFO' database and CAS, and
Dovepress

is the official journal of The International Neuropsychiatric Association (INA). The manuscript management system is completely online and includes a very quick and fair peer-review system, which is all easy to use. Visit http://www.dovepress.com/testimonials.php to read real quotes from published authors. 